\title{
On a doped transition layer in the space charge region of Schottky contact
}

\author{
L.V. Shekhovtsov \\ Institute of Semiconductor Physics, NAS of Ukraine, 45 prospect Nauky, 03028 Kyiv, Ukraine \\ Phone: 38(044) 265 6272; fax: 38(044) 265 8342; e-mail: k208@photon.kiev.ua
}

\begin{abstract}
A technique is proposed for estimation of maximal thickness of a doped semiconductor transition layer in a Schottky contact. It is based on taking spectral curves of transverse photovoltage. It is shown that in gallium arsenide crystal with starting impurity concentration of $10^{16} \mathrm{~cm}^{-3}$, a layer with doping level of $10^{17} \mathrm{~cm}^{-3}$ and maximal thickness of $4 \mathrm{~nm}$ may exist in the space-charge region near the interface of the $\mathrm{TiB}_{2}-\mathrm{GaAs}$ structure. At impurity concentration in the transition layer about and over $10^{18} \mathrm{~cm}^{-3}$, its thickness goes down, and it shows up as individual inclusions in the GaAs lattice. The total area of such inclusions at the metal-semiconductor interface does not exceed $1 \%$ that of contact. In actual structures the transition layer is a superposition of layers and inclusions, with impurity concentration of $10^{17} \mathrm{~cm}^{-3}$ or more and thickness below $4 \mathrm{~nm}$.
\end{abstract}

Keywords: Schottky contact, transition layer, transverse photovoltage, space-charge region, depletion region.

Paper received 08.11.02; accepted for publication 17.12.02.

\section{Introduction}

Appearance of a doped transition layer in semiconductor crystal at metal-semiconductor contact formation may change (and impair) the electrophysical parameters of various device structures. Such layers have been observed, for instance, in model samples of $\mathrm{TiB}_{2}-\mathrm{GaAs}$ and $\mathrm{Au}-$ $\mathrm{TiB}_{2}-\mathrm{GaAs}[1]$ and Au-GaAs [2] Schottky contacts.

One needs to estimate thickness and doping level of such layer, especially in the cases when direct (or destructive) techniques cannot be applied. Indirect techniques (that are based on approximations) are characterized by a certain limit of accuracy [3,4]. They do not enable one to determine dopant concentration changes below the above limiting value, or establish presence of a layer whose thickness is less than the Debye shielding length.

The problem of estimation of maximum possible thickness of a doped layer is urgent, first of all, for correct interpretation of the results of photoelectric measurements made for these structures.

\section{Results and discussion}

Presence of a doped transition layer in GaAs has been established using different experimental techniques [1, $2,5,6]$. In particular, the results of measurements of spectral curves for transverse photovoltage in the $\mathrm{TiB}_{2}-\mathrm{GaAs}$ and $\mathrm{Au}-\mathrm{TiB}_{2}-\mathrm{GaAs}$ Schottky contact samples indicated at formation of such layer [1].

If a doped layer appears near interface, then the light absorption edge of semiconductor crystal shifts toward lower energies due to formation of state density "tails". When doping results from diffusion of atoms from a metal film, one may expect that the doping level of the transition layer would be inversely related to the layer thickness. An actual dopant depth profile is affected by various diffusion mechanisms [5].

Thus one can estimate transition layer thickness reasoning from the following assumptions: (i) the maximal thickness $d$ of a doped semiconductor layer is below the space-charge region (SCR) width $L_{D}$, (ii) the doping level of transition layer decreases when going from the interface into the crystal bulk, and (iii) there is no dopant concentration gradient along the interface. 


\section{L.V. Shekhovtsov: On a doped transition layer in the space ...}

In what follows, we shall use such symbols: $L$ is the width of depletion region of Schottky contact; $N$ is the dopant concentration in the transition layer; $\left(N_{D}-N_{A}\right)$ is the starting dopant concentration in the semiconductor bulk and depletion region $L ; \Delta\left(N_{D}-N_{A}\right)$ is the dopant concentration change in the depletion region; $S$ is the metal-semiconductor contact area.

If additional doping occurs due to transition layer formation only, then the total number of dopant atoms in it is equal to the change of dopant atoms number in the whole depletion region $L$ :

$S \times d \times N=S \times L \times \Delta\left(N_{D}-N_{A}\right)$.

Then

$$
d=\left[\Delta\left(N_{D}-N_{A}\right) / N\right] \times L .
$$

On the other hand, the total number of dopant atoms in SCR is also equal to the change of dopant atoms number in the depletion region:

$$
S \times L_{D} \times\left(N_{D}-N_{A}\right)=S \times L \times \Delta\left(N_{D}-N_{A}\right) .
$$

Therefore,

$$
\Delta\left(N_{D}-N_{A}\right)=L_{D} \times\left(N_{D}-N_{A}\right) / L .
$$

Substituting expression (4) in (2), one obtains:

$$
d=\left[\left(N_{D}-N_{A}\right) / N\right] \times L_{D}
$$

For GaAs with doping level of $10^{16} \mathrm{~cm}^{-3}$, the Debye shielding length $L_{D}$ is $40 \mathrm{~nm}$ [7].

A comprehensive analysis of the features of transverse photovoltage spectral curves taken for the $\mathrm{TiB}_{2}-\mathrm{GaAs}$ structure samples was made in [1]. Shown in Fig. 1 are the spectral curves taken for two such samples, non-annealed and annealed at a temperature $T=400{ }^{\circ} \mathrm{C}$. The shift of the photovoltage spectral curve edge due to appearance of state density "tails" corresponds to the doping level of the transition layer of about $10^{17} \mathrm{~cm}^{-3}$. Then the transition layer thickness $d$ estimated from expression (5) is $4 \mathrm{~nm}$.

If the dopant concentration in the transition layer is $10^{18} \mathrm{~cm}^{-3}$, then its maximal thickness is to be $0.4 \mathrm{~nm}$. This value is less than the GaAs lattice constant, and so it is physically non-correct. But this result followed from the assumption on presence of a uniform transition layer along the interface. It is evident that at impurity concentration over $10^{18} \mathrm{~cm}^{-3}$ the doped layer is made of discrete inclusions in the GaAs lattice.

Thus, a layer with doping level of $10^{19} \mathrm{~cm}^{-3}$ may be $4 \mathrm{~nm}$ thick (this value is equal to the thickness of a continuous layer with doping level of $10^{17} \mathrm{~cm}^{-3}$ ), if its total area at the GaAs crystal surface contacting with the metal film is below $1 \%$. Decrease of this area corresponds to increase of thickness of discrete inclusions with such concentration. However, as it follows from the above starting assumptions, this thickness value should be less than the Debye shielding length $L_{D}$.

It should be noted that generally a transition layer is a superposition of the near-contact regions with different doping levels. Some regions with higher impurity concentrations exist against the background of a layer whose doping level is, say, $10^{17} \mathrm{~cm}^{-3}$. And, respectively, such layer thickness will vary along the interface, and its total thickness is to be less than that of a layer whose doping level is constant. Shown in Fig. 2 is a possible model for such transition layer.

At thermal annealing of metal-semiconductor structures impurity concentration at the interface may also change due to their passivation and gettering from the bulk. This mechanism may become not only competing, but even predominant [5]. If thickness of such layer near

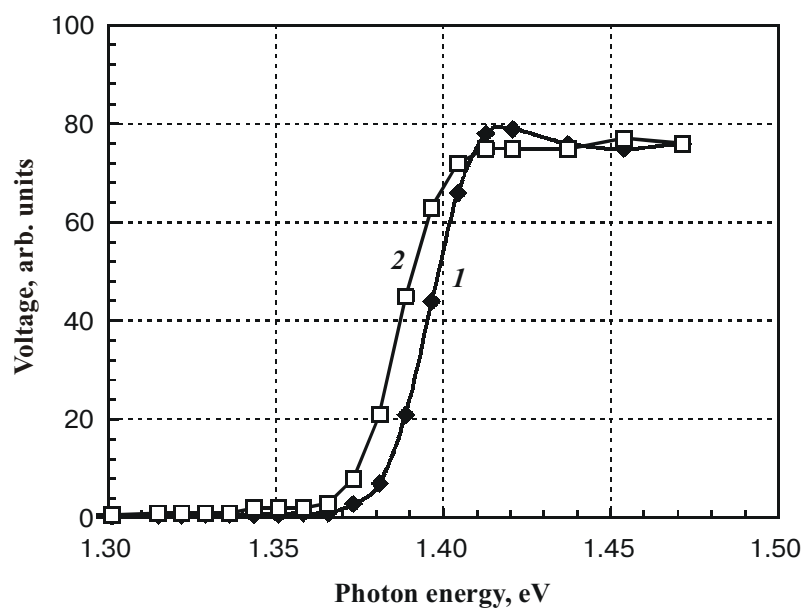

Fig. 1. Spectral curves taken for two $\mathrm{TiB}_{2}-\mathrm{GaAs}$ structure samples: 1 - non-annealed, $2-$ annealed at $T=400{ }^{\circ} \mathrm{C}$.

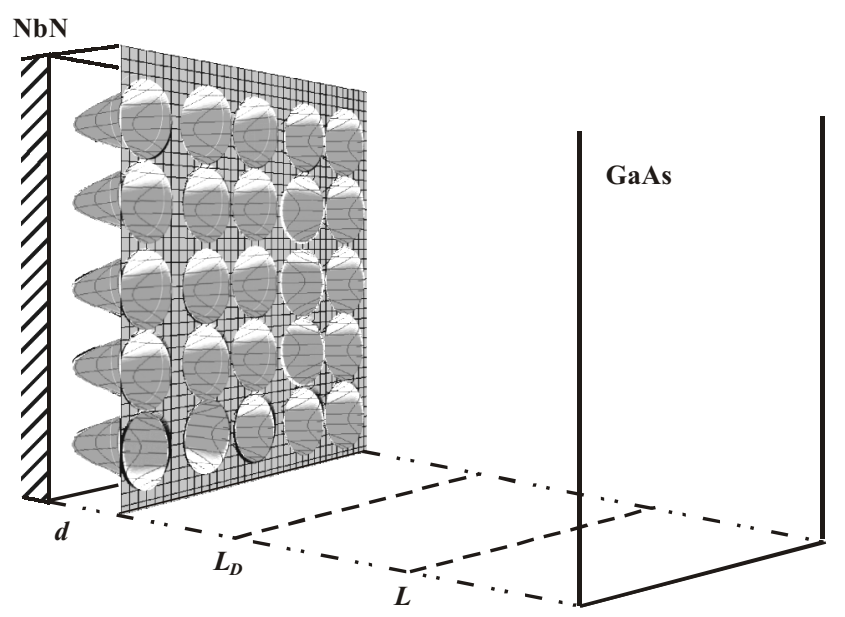

Fig. 2. Model for doped transition layer in Schottky contact SCR. $d$-transition layer; $L_{d}$ - space-charge region; $L-$ depletion region. 


\section{L.V. Shekhovtsov: On a doped transition layer in the space ...}

the interface is over the Debye shielding length $L_{D}$, then one can measure the impurity concentration in it using, for instance, the $C$ - $V$ curves technique [7]. And if its thickness is below $L_{D}$, then the above considerations are still valid.

It was stated earlier that the indirect methods for measurement of dopant distribution in a Schottky contact have intrinsic limitation of resolution. This is related, first of all, to inaccuracies of the contact area $(\sim 0.5 \%)$ and $\Delta C / C$ (less than $1 \%$ ) measurements. Besides, in the $C$ - $V$ curves technique presence of an abrupt boundary between the depletion and quasi-neutral regions of a sample is presumed. The free charge carrier concentration is measured at the depletion region edge where inaccuracy is maximal, because the methodical approach itself is based on the assumption that the free charge carrier concentration at a given depth is that of dopant. If these rather crude approximations are not taken into account, then one may take the limit to resolution of this technique to be $1 \%$. In other words, it is impossible to determine impurity concentration changes in the depletion region that are below the above level.

On the other hand, if variation of the impurity concentration in the depletion region (or, rather, in SCR) of the Schottky contact lies within the above resolution value and is related to the transition layer formation, then its maximal thickness (found from the above expressions) is $0.01 \mathrm{~L}$, i.e., $4 \mathrm{~nm}$. And the analysis presented above gives just the same value for the maximal thickness of the transition layer in the case when the doping level in SCR is $10^{17} \mathrm{~cm}^{-3}$. This means that the limitation put by the limiting resolution of the $C$ - $V$ curves technique on determination of dopant concentration in the near-contact region is such that its value does not exceed that due to shielding of the external electric field in the semiconductor bulk.

Therefore, the assumption (made before the above calculations of the transition layer thickness) that this thickness does not exceed the SCR width is quite reasonable. In other words, the principal limitation for the $C-V$ curves technique is provided by shielding of the external field in semiconductor.

\section{Conclusion}

From the above it follows that estimation of the transition layer thickness is possible. It is based on the assumption that variation of the doping level of semiconductor occurs in the depletion region of the Schottky contact due to increase of the dopant concentration in the transition layer whose thickness $d$ is below the Debye shielding length in GaAs.

Reasoning from the experimentally observed shift of the transverse photovoltage spectral curve edge towards lower energies [1], one can conclude that in $\mathrm{TiB}_{2}-\mathrm{GaAs}$ structure a transition layer exists whose maximal thickness is $4 \mathrm{~nm}$ and uniform doping level is $10^{17} \mathrm{~cm}^{-3}$. A transition layer with impurity concentration $10^{18} \mathrm{~cm}^{-3}$ and more can exist in such structures as discrete inclusions in $\mathrm{GaAs}$ semiconductor crystal. The total area of these inclusions at the metal-semiconductor interface is less than $1 \%$ total contact area. It seems that in actual structures the transition layer is a superposition of layers and inclusions with different doping levels.

It should be noted in closing that determination of doped transition layer thickness using other techniques gave the results that are close to the obtained above [8].

\section{References}

1. E.F. Venger, R.V. Konakova, O.B. Okhrimenko, S.Yu. Sapko, L.V. Shekhovtsov, V.N. Ivanov, A transitional layer in $\mathrm{TiB}_{2}-$ GaAs and $\mathrm{Au}-\mathrm{TiB}_{2}-\mathrm{GaAs}$ Schottky contacts // Fiz. Tekh. Poluprov. 35(4), pp. 439-444 (2001) (in Russian).

2. B.I. Bednyi, On transformation of the potential barrier at GaAs/ Au interface during thermal treatment // Fiz. Tekh. Poluprov. 33(11), pp. 1350-1354 (1999) (in Russian).

3. I.R. Karetnikova, I.M.Nefedov, and V.I.Shashkin,, On the accuracy of the semiconductor doping profile extraction on the basis of $\mathrm{C}-\mathrm{V}$ measurements in the process of elektrochemical etching // Fiz. Tekh. Poluprov. 35(7), pp. 801-807 (2001) (in Russian).

4. V.V. Batavin, Control of Parameters of Semiconductor Materials and Epitaxial Structures, Sovetskoe Radio, Moscow (1976) (in Russian)

5. A.N. Andronov, N.T.Bagraev, L.E.Klyachkin, and S.V.Robozerov, et al., Ultrashallow $\mathrm{p}^{+}-\mathrm{n}$ junctions in silicon (100): electron-beam diagnostics of sub-surface region // Fiz. Tekh. Poluprov. 32(2), pp. 137-143 (1998) (in Russian).

6. V.G. Bozhkov, V.A.Kagadey, and N.A.Torkhov, Effect of atomic hydrogen on the properties of Schottky-barrier metalGaAs contacts // Izv. Vuzov. Fizika No 8, pp. 115-121 (1997) (in Russian).

7. S.M. Sze, Physics of Semiconductor Devices, $2^{\text {nd }}$ edition, John Wiley and Sons, Inc., New York. Chichester. Brisbane. Toronto. Singapore (1981).

8. Z. Liliental-Weber, R.Gronsky, J.Washburn, N.Newman, W.E.Spicer, and E.R.Weber, Schottky and Ohmic Au contacts on GaAs: Microscopic and electrical investigation. // J. Vac. Sci. Technol. B4(4), pp. 912-918 (1986). 